\title{
Application of Nano-Composites in the Recovery of Sports Ligament Injury
}

\author{
C. J. XIE AND LEILEI TIAN*
}

College of Physical Education, Jingdezhen University, ${ }^{1}$ Teaching Department of Physical and Military Education, Jingdezhen Ceramic Institute, Jingdezhen 333000, China

Xie et al.: Application of Nano-Composites in the Ligament Injury

\begin{abstract}
To study the application of nano-composites in the recovery of sports ligament injury is the main objective. Rabbits were divided into two groups on average. Firstly, cell samples were prepared after circumcision in children and nano-composite scaffolds were prepared by electrospinning driven by current. Cell samples were planted and cultured on nano-composite scaffolds to prepare nano-composite scaffolds mimicking extracellular matrix. The surgical sutures of the scaffolds were embedded in the injured motor ligaments. In the anterior cruciate ligament of the experimental sample, the experimental group was set up; in the control group, the experimental sample only received basic nursing care. At the $2^{\text {nd }} \mathbf{w}$ after operation, the tissue edema around the incision of knee joint was observed in the experimental group, no absorption of micro suture was observed, the gap between tendon and bone tunnel was obvious, the anterior cruciate ligament and tibia and femur bone were loose; at the $4^{\text {th }} \mathrm{w}$ after operation, the antibacterial micro suture partially absorbed the tissue edema of knee joint, there was no obvious gap between tendon-bone tunnel interface and anterior cruciate ligament. At the $8^{\text {th }} \mathbf{w}$ after operation, the edema of knee joint completely disappeared, the interface between tendon and bone tunnel was well repaired, most of the anti-bacterial micro sutures were absorbed and the anterior cruciate ligament and cancellous bone at the tibiofemoral junction were tightly bonded. Stem cell nano-composite fiber scaffolds can enhance the bonding strength of tendon-bone tunnel interface, promote the repair of tendon-bone tunnel interface and improve the maximum stiffness and tensile strength of tendon-bone tunnel interface in the experimental group, which indicates that stem cell nano-composite fiber scaffolds are active and effective in restoring injured ligaments.
\end{abstract}

Key words: Nano-composites, composite material, ligament injury, tendon

Anterior cruciate ligament (ACL) injury is the main cause of knee pain and dysfunction. It is one of the most common musculoskeletal injuries in the field of sports medicine, accounting for about $20 \%$-35 \% of knee joint diseases, which seriously affects the life and work of patients. At present, the clinical treatment of tendon injury mainly includes physiotherapy, surgical suture and tissue transplantation. Micro suture is a sterile, nylon monofilament surgical suture that is used for general soft tissue. However, the histomorphology and functional characteristics of tendon after repair are far from those of normal tendon and their learning performance is poor, accompanied by tendon withering and sore tissue formation ${ }^{[1]}$. Therefore, the means of tendon regeneration and repair need to be developed urgently. When mechanical action is transmitted between relatively hard, non-retractable tissues and relatively soft, retractable tissues, the bonding position often becomes a weak point, which leads to the concentrated force on the corresponding parts. The objective of reconstruction of ACL injury is to restore the normal composite structure of tendon-bone tunnel interface ${ }^{[2]}$. Some studies have found that in the process of ligament repair, the tendon-bone junction complex is a gradual maturation process. Because of the special structure and complexity of the tendon-bone tunnel interface, early and mid-term reconstruction techniques cannot guarantee the tendon to heal firmly in the bone marrow canal. Postoperative complications such as re-avulsion failure cannot be completely avoided.

*Address for correspondence

E-mail: tianleilei0727@163.com 
Therefore, new treatment methods need to be further explored $^{[3,4]}$.

Regarding the treatment of ACL injury, clinical functional training after operation can effectively promote the repair of soft tissue injury, including the repair of ligaments and tendons. However, redislocation and secondary instability often occur as clinical complications. It is of great significance to clarify the biomechanical characteristics and mechanical environment of ACL tendon-bone structure for tendon attachment repair. In many previous experiments, the effects of tendons and ligaments in normal tendons and ligaments and in healing process have been evaluated, but there is no relevant study on the bonding strength and time-intensity curve of tendon-bone attachment ${ }^{[5-7]}$. In order to elucidate its histological and biological healing mechanism, it is necessary not only to analyze the characteristics of tissue structure, but also to analyze the situation of tendon growing into bone tunnel at the interface of tendon-bone tunnel. The quasi-linear viscoelastic (QLV) model, originally established by Fung, can measure the viscous and elastic parameters in the pressure relief experiment and can quantitatively analyze the nature of tendon attachment points in the healing process. It has been successfully applied in many fields. Soft tissue research, but not yet applied to normal and healing tendon attachment research ${ }^{[8,9]}$.

In summary, this paper combines nano-composite materials with stem cell culture technology in vitro to study the recovery of sports ligament injury. Stem cell culture technology in vitro constructs bio-inductive materials imitating extracellular matrix, which can effectively regulate the proliferation and differentiation of stem cells, repair the mechanical properties of tendon ${ }^{[10]}$, promote the differentiation of tendon stem cells into tendon system and combine them. The scaffolds made of nano-composites can enhance the regeneration of tendons in vivo.

\section{MATERIALS AND METHODS}

\section{Materials and instruments:}

Reagents needed: Dulbecco's modified eagle medium (DMEM) series of cell culture medium, serum substitutes, phosphate buffered saline (PBS), prolase, $100 \mathrm{U} / \mathrm{ml}$ penicillin/mitomycin (double antibody), nonessential amino acids, L-glutamine, prolactin-releasing hormone (PRH), ascorbic acid-2-phosphate salt, vinyl chloride (VC) (Sigma), gelatin (Sigma) basic fibroblast growth factor (bFGF), hematoxylin-eosin (HE) staining solution, polymethyl methacrylate (PCP) Trichostatin
A (TSA).

Cell culture related instruments: Centrifuge (Eppendorf), inverted fluorescence electron microscopy (Olympus), etc. Other instruments: Hitachi, Beckman Coulter, Olympus, Quanta 10 FEI, Instron, automatic dyeing machine, etc.

Samples: 24 female rabbits were injured by driving exercise.

\section{Experimental methods:}

Isolation and culture of stem cells, human foreskin fibroblast (HFF): Fresh prepuce specimens from children after circumcision were soaked in high-sugar medium for transportation. PBS is cleaned five times and soaked in alcohol for 5 min to prevent contamination.

PBS was washed 2-3 times to separate subcutaneous fat and loose connective tissue. Wash PBS 2-3 times and cut into small pieces. $0.025 \%$ collagenase I was digested in a refrigerator at $4^{\circ}$ for about $13-18 \mathrm{~h}$ and the epidermis and dermis were separated. Cut the dermis into pieces about $1 \mathrm{~mm}$ in size, the smaller the better. Wash the dermis 3-5 times with PBS. Cell culture plates were moved to $10 \mathrm{~cm}$ to distribute tissue blocks evenly. Tissue blocks were evenly planted on the culture plates with photographs, no interval of $5 \mathrm{~mm}$ or so, no plate was inoculated with 30 or so. The high sugar medium (containing $20 \%$ fetal bovine serum (FBS)) was added about $3 \mathrm{ml}$ and then incubated in $37^{\circ}, 5 \% \mathrm{CO}_{2}$ incubator. 2-3 d later, when it proliferates to about 70 $\%$, it can be passed on. PBS was cleaned once, digested by $0.05 \%$ trypsin, neutralized in a suitable amount of medium, $300 \mathrm{~g}$ from $5 \mathrm{~min}$ and cultured in $37^{\circ}, 5 \%$ $\mathrm{CO}_{2}$ incubator after suspension of fresh medium.

Preparation of nano-fiber scaffolds: Ultrafine parallel nanofibers were prepared by current-driven electrospinning that is stable jet electrospinning (SJES). The overall preparation methods are as follows: chitosan $(\mathrm{CTS}$, molecular weight $(\mathrm{Mw})=900000 \mathrm{Da})$ and gelatin (GT) was dissolved in trichloroacetic acid (TFA) at a weight ratio of $6: 1$ to prepare solution 1; poly-L-lactic acid (PLLA, Mw=100 $000 \mathrm{Da}$ ) and polyoxyethylene $(\mathrm{PEO}, \mathrm{Mw}>5000000 \mathrm{Da})$ at a weight ratio of 3:1. The ratio is dissolved in dichloromethane to prepare solution 2. After mixing solution 1 and 2 , a mixture of chitosan $(62.1 \%, 20.7 \%, 10.3 \%$ and $6.9 \%$ of CTS, PLLA, GT and PEO, respectively) was obtained as the electrospinning solution of SJES. The disordered nano fibers were prepared by conventional electrospinning (CES) and the composition of electrospinning solution was the same as that of SJES. Injection pump (KDS100) 
and high voltage power supply (TXR1020N30-30, Teslaman) are used to control the flow rate and voltage of electrospinning fluid during electrospinning process, respectively. Parallel and disordered spinning chips were obtained after electrospinning for $2 \mathrm{~h}$. The thickness ranged from $0.11 \mathrm{~mm}$ to $0.12 \mathrm{~mm}$. The synthesized spinning chips were then immersed in 0.5 $\mathrm{ml}$ sodium hydroxide solution for $30 \mathrm{~min}$ and ethanol was washed three times. The obtained nano-fibers scaffolds were transferred to cover slides and used for overnight sterilization with ethanol and ultraviolet before cell culture.

Cell culture on nano-fiber scaffolds: The bracket was cut into $1.4 \mathrm{~cm} \times 1.4 \mathrm{~cm}$ size, treated with $75 \%$ alcohol for $2.5 \mathrm{~h}$, cleaned by PBS and overnight irradiated by ultraviolet light. A plate of $10 \mathrm{~cm}$ diameter culture dish full of cells was taken and washed with PBS. 1 $\mathrm{ml}$ of trypsin was digested for $2 \mathrm{~min}$. The medium was neutralized in equal volume. Transfer to 15 $\mathrm{ml}$ centrifugal tube, $1200 \mathrm{rpm}, 5 \mathrm{~min}$. Remove the supernatant, add $1 \mathrm{ml}$ medium to suspend the cells and add $2 \times 10^{4}$ cells $/ \mathrm{cm}^{2}$ density to the material. After incubation at $37^{\circ}$ for $2 \mathrm{~h}$, the medium was added to the corresponding amount. TSA was added into nanofibers scaffolds to prepare four kinds of TSA modified/ unmodified parallel/disordered nano-fibers scaffolds: A nano-fibers scaffolds, A-TSA nano-fibers scaffolds, $\mathrm{R}$ nano-fibers scaffolds and R-thrombospondin (TSP) R-TSPs nano-fibers scaffolds.

Cell proliferation assay: In 12-well plate, the initial density of cells was $2 \times 10^{4} / \mathrm{cm}^{2}$. At each time point, $400 \mathrm{ml}$ cell counting kit-8 (CCK-8) medium was added to the cornea and incubated in $37^{\circ}$ and $5 \% \mathrm{CO}_{2}$ humidity incubator for 1-4 $\mathrm{h}$. The optical density (OD) value was read by enzyme-labeled instrument and cell proliferation was calculated.

Scanning electron microscope observation of cell growth and morphology on material: Human induced pluripotent stem cell-derived mesenchymal stem cells (HiPSC-MSCs) were implanted on nanofiber scaffolds at a concentration of $2 \times 10^{4} / \mathrm{cm}^{2} .3 \mathrm{~d}$ after planting, the morphology and distribution of cells could be observed by scanning electron microscopy. Samples were immobilized in $0.25 \%$ dialdehyde solution and then washed with PBS three times for $30 \mathrm{~min}$. The samples were fixed in osmium acid for $40 \mathrm{~min}$, then rinsed with PBS three times for $30 \mathrm{~min}$ and then dehydrated in acetone with increasing concentration $(30-100 \% \mathrm{v} / \mathrm{v})$. After drying, the samples were buried and coated with gold in the aluminum tube and then observed at an accelerating voltage of $15 \mathrm{kV}$.

\section{Statistical analysis:}

All data are expressed as mean \pm standard deviation (SD), n (>3). The collagen content data were analyzed by statistical package for the social sciences (SPSS) 25 . The difference of gene expression level was tested by $\mathrm{t}$-test. The significant difference was defined as $\mathrm{p}<0.05$, expressed as *; $\mathrm{p}<0.01$, not $* * ; \mathrm{p}<0.001$, expressed as $* * *$.

\section{Construction of the model after ACL reconstruction:}

According to the standard operation methods of ACL reconstruction in practical orthopedics and orthopedics surgery and combined with the requirements of surgical operation in medical experimental zoology, the operation methods of ACL reconstruction after injury in this experiment were formulated. In this experiment, the ACL of knee joint was reconstructed by tunnel method and suture method. The plaster was fixed for $1 \mathrm{w}$ and removed after $1 \mathrm{w}$. The repair mechanism of tendon-bone tunnel interface and deep bone site after ACL reconstruction was studied and the condition of each index at different time stages and different training methods was observed, so that the detection results of the index were phased and dynamic ${ }^{[1]}$.

After cleaning the animal operating table, rabbits were bound and fixed, rabbit hair was removed from both knees by electric razor, cleaned with soapy water, routine iodophor disinfection towel was used and lidocaine hydrochloride local infiltration anesthesia was used. The anterior longitudinal incision of the knee was taken to cut the skin and subcutaneous tissue directly, expose and incise the structure of the expanded ligament near the patella, expose the attachment point of the ACL to the tibiofemoral joint, cut the attachment point of the ACL between the tibial plateau and the femoral condyle with a scalpel and make a bone groove with an electric drill at the attachment point of the tibiofemoral joint ${ }^{[12]}$. Rabbit Achilles tendon was reconstructed and fixed. In the experimental group, stem cell nano-composite fibrous scaffolds were installed in the bone groove and the Achilles tendon was cut in advance for mattress suture preparation. The distal part was sutured into the bone groove, layer by layer and the sterile dressing bandage was bandaged. After operation, the knees were fixed with plaster bracket for $1 \mathrm{w}$. During the period of plaster fixation, artificial supplementary diet was given according to the situation. After operation, the prophylactic antibiotics were injected into 400000 units of muscle according to the body weight of experimental 
animals, once a day and $3 \mathrm{~d}$ before operation. After the removal of plaster external fixation of bilateral knee joints, the experimental animals gradually recovered from artificial rearing to the level of independent diet. The control group was not implanted with nano-fibers scaffolds.

Two normal rabbits were randomly executed by computer random number method and bilateral knee joint specimens including tendon-bone tunnel interface (including tibia, ACL and femur) were taken out. Routine fixation, decalcification, slicing and staining were used as normal indicators for control. The experimental group and the control group were executed with excessive anesthetics at 2, 4 and $8 \mathrm{w}$ after operation. The knee joint samples were obtained by the same method. The soft tissue around the knee joint was removed and tibia, ACL and femur were consecutively preserved ${ }^{[13]}$. The tendon-bone tunnel interface of the knee joint can be preserved, but the tibia-femur, ACL and their junctions should be included for histological and immunohistochemical observation. After 4-5 w of decalcification, the specimens were fixed with polyformaldehyde solution and washed (ethylenediamine tetraacetic acid disodium salt decalcification solution), then the decalcification solution was washed with running water for $30 \mathrm{~min}$.

After $30 \mathrm{~s}$ of differentiation, ethanol hydrochloride was washed with tap water for $10 \mathrm{~min}$, hematoxylin stained for $5 \mathrm{~min}$ and distilled water washed for $2 \mathrm{~s}$ to remove excess dye. After dyeing with eosin dye for $1 \mathrm{~min}$, slices should be cut in order to prevent overdyeing for $2 \mathrm{~min}, 95 \%$. Ethanol was treated for $5 \mathrm{~s}$ and distilled water was used to remove the excess dye.

\section{RESULTS AND DISCUSSION}

Observation results of experimental animals and cut specimens were described here. At the $1^{\text {st }} \mathrm{w}$ after the removal of plaster, there was one side in the excluded group ( 1 case of knee joint infection) and 18 rabbits in the control group were selected for analysis. After the operation, the incisions of the other two groups healed at the first stage and there were no complications such as swelling, infection and purulence. There was no abnormal feeding process in the animals. At the $2^{\text {nd }} \mathrm{W}$ after operation, the tissue edema around the incision of knee joint was observed in the experimental group and no absorption of micro suture was observed. The gap between tendon and bone tunnel was obvious. The ACL and tibia-femur bone joint were loosened. At the $4^{\text {th }} \mathrm{w}$ after operation, the anti-bacterial micro suture was partially absorbed in the experimental group and the knee joint tissue edema was significantly reduced. There was no obvious gap between tendon-bone tunnel interface and ACL and tibia. At the $8^{\text {th }} \mathrm{W}$ after operation, the edema of knee joint completely disappeared, the interface between tendon and bone tunnel was repaired well, most of the anti-bacterial micro sutures were absorbed and the ACL and cancellous bone at the tibiofemoral junction were tightly bonded.

Immunohistochemical observation and HE staining were used to observe histomorphology. In the experimental group, the expression of antibody staining was negative (fig. 1A) in the sports injured ligaments repaired with A nano-fibers scaffolds. HE staining showed that typical tendons and bone tissues were intercalated and intersected tightly. Bone trabeculae and tendon fibers were arranged orderly and tightly (fig. 1B).
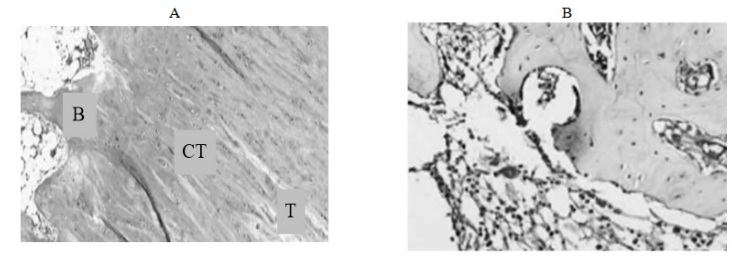

Fig. 1: (A) Histomorphology of rabbit normal and (B) expression of experimental group of rabbit normal ACL tendon-bone tissue under light microscope, diaminobenzidine (DAB) x200

At the $2^{\text {nd }} \mathrm{W}$ after operation, the experimental group was mainly composed of inflammatory granulation tissue and tendon-bone cell growth was rare. In the control group, HE staining showed that the width of tendonbone tunnel interface was wider, the width of tendonbone tunnel interface was wider and the distribution was uneven (fig. 2A and fig. 2B).
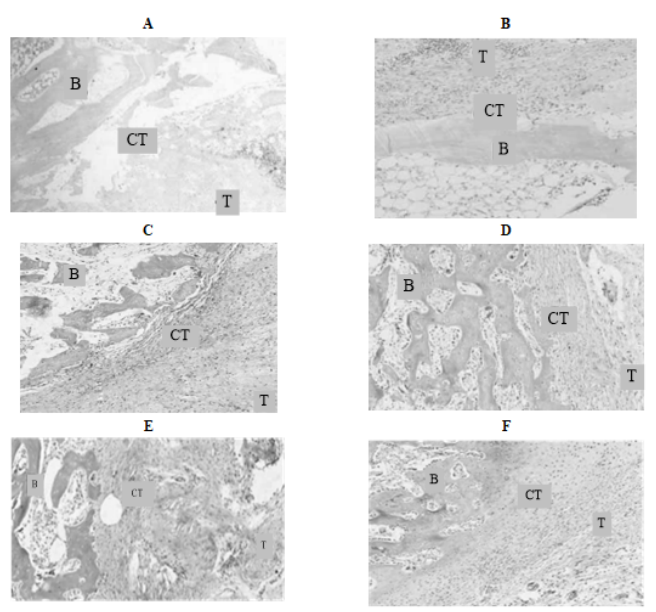

Fig. 2: Histomorphology of ACL tendon-bone tissue under light microscope, HE x100. (A) Control group $2 \mathrm{w}$ after operation; (B) experimental group $2 \mathrm{w}$ after operation; (C) control group 4 $\mathrm{w}$ after operation; (D) experimental group $4 \mathrm{w}$ after operation; (E) control group $8 \mathrm{w}$ after operation; (F) experimental group $8 \mathrm{w}$ after operation 
At the $4^{\text {th }} \mathrm{W}$ after operation, the tissue structure of the experimental group was more compact and the fibrocyte increased, forming a structure similar to the direct stop point. In the control group, the tendon-bone tunnel interface, a large number of fibroblast inflammatory granulation tissue was excessive to tendinous tissue and the gap width was uneven and reduced. In the control group, HE staining showed a decrease of inflammatory cells at the tendon-bone tunnel interface (fig. $2 \mathrm{C}$ and fig. 2D).

Control group $8^{\text {th }} \mathrm{W}$ after operation and experimental group $8^{\text {th }} \mathrm{W}$ after operation was shown in fig. $2 \mathrm{E}$ and fig. 2F.

In the control group, the antibody staining was shallow, while in the experimental group, the expression of bone morphogenetic protein 2 (BMP-2) antibody was positive, the staining was obvious and the staining was more uniform (fig. 3A and fig. 3B).
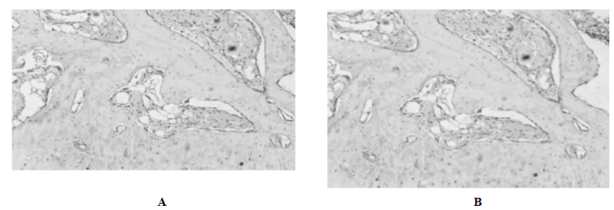

Fig. 3: The expression of BMP-2 of ACL tendon-bone under light microscope, DAB x100. (A) Control group $4 \mathrm{w}$ after operation and (B) experimental group $2 \mathrm{w}$ after operation

Morphology and proliferation of tendon stem cells on four kinds of nano-fibers were described here. With Scleraxis (Scx)-green fluorescent protein (GFP) (ScxGFP) tendon stem cell reporting system, we observed the effects of four kinds of nano-fiber scaffolds on cell morphology, proliferation and Scx expression. The results showed that the oval nuclei of tendon stem cells on the A-TSA scaffold were aligned in the direction of nano-fibers. Most of the tendon stem cells grown on the A-TSA nano-fibers were Scx-GFP-positive and only a few Scx-GFP-positive cells were observed on the nanofiber A. The nuclei of tendon stem cells on scaffold $\mathrm{R}$ were round and undirected. Scx-GFP was positive in a small part of tendon stem cells grown on nano-fiber R-TSA, while Scx-GFP was negative in the cells grown on nano-fiber R. Quantitative results of translucent cells are consistent with those described above (fig. 4). It shows that TSA modified parallel nano-fibers can not only maintain the long spindle shape of muscle knee stem cells, but also stimulate the expression of Scx, which is more conducive to the differentiation of tendon system. The cell density on the scaffolds at w 2, 4 and 8 was compared to detect the cleavage and proliferation of muscle wax stem cells. There was no statistical difference (fig. 5).

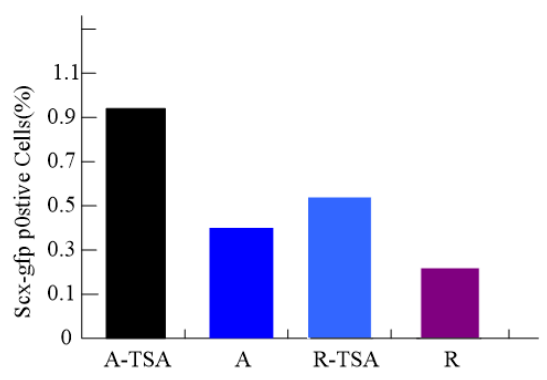

Fig. 4: The Scx-GFP tendon stem cells grew GFP positive cell rates on four types of scaffolds

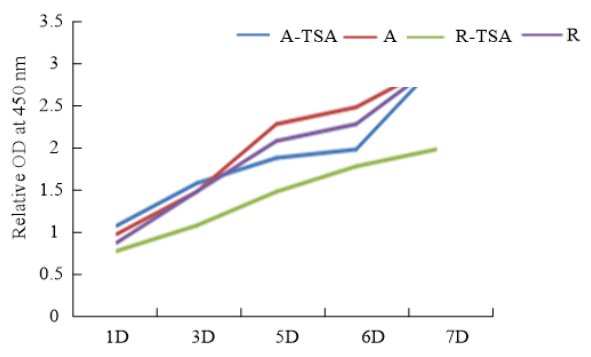

Fig. 5: Growth curves of tendon stem/progenitor cells (TSPCs), (一) A-TSA, (一) A; (-) R-TSA; ( - ) R

In order to study the regulatory effects of four kinds of scaffolds on the differentiation of TSPs tendon, we examined the expression of tendon-related genes (Scx, Mohawk Homeobox (MKX), eyes absent homolog 1/2 (EYA1/2), sine oculis homeobox homolog 2 (SIX2), Homeobox A11 (HOXA11) and early growth response protein 1 (EGR1)) after TSPs were grown on different scaffolds and compared with the cultured tendon stem cells on the plate. The results showed that the expression of tendon-related genes of tendon stem cells grown on scaffolds A-TSA or A was significantly up-regulated compared with that of tendon-related genes of tendon stem cells grown on flat plates. Moreover, A-TSA promoted the expression of tendon-related genes more than A scaffold, especially EGR1 $(\mathrm{p}<0.05)$, SIX2 ( $\mathrm{p}$ $<0.05)$, HOXA11 $(\mathrm{p}<0.01)$. R-TSPs scaffold did not promote the expression of tendon-related genes in TSPs compared with control group, while R-TSPs scaffold increased the expression of tendon genes in TSPs and the effect was similar to that of scaffold A (fig. 6).

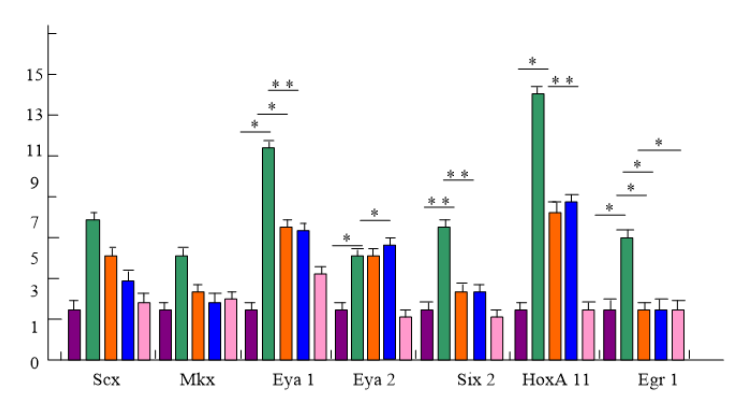

Fig. 6: Messenger RNA expression levels of tendon-related genes after TSPs implantation on scaffolds for $3 \mathbf{d}$; ${ }^{*} \mathbf{p}<\mathbf{0 . 0 5}$, **p $<$ 0.01, Con ( $\square$ ); A-TSA ( $\square$ ); A ( $\square$ ); R-TSA ( $\square$ ); R( $\square$ ) 
Subsequently, we compared the expression levels of tendon differentiation-related proteins by immunofluorescence and further compared the effects of four scaffolds on the tendon differentiation ability of tendon stem cells (fig. 7). Consistent with the results of gene detection, A-TSA has the most significant effect on promoting the differentiation of tendon stem cells in the four scaffolds. On the basis of increasing the expression of Scx in the nucleus, it also significantly enhanced the expression of collagen (COLL), collagen 5 (COL5) and Ephrin type-A receptor 4 precursor (EPHA4).

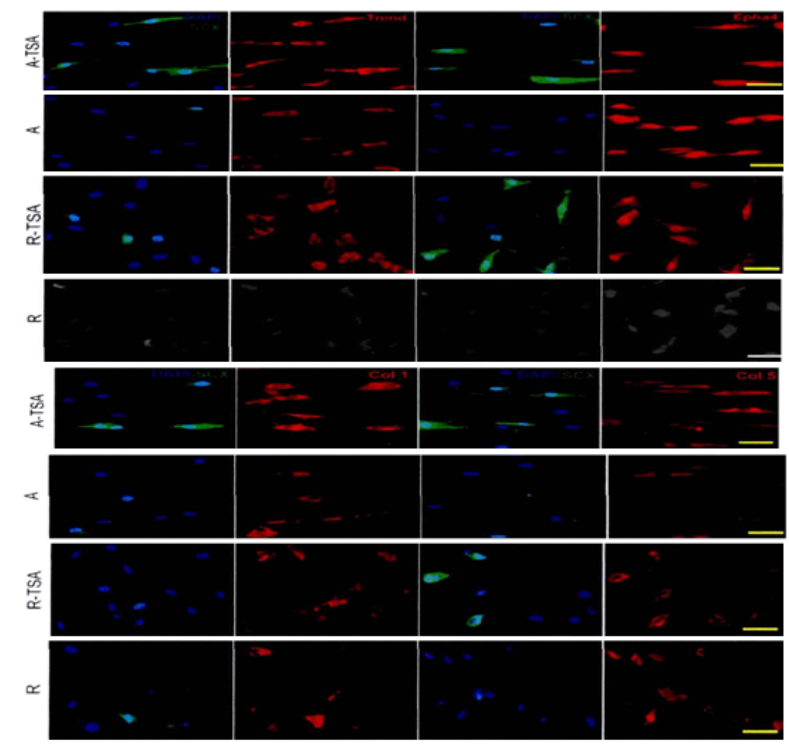

Fig. 7: Immunofluorescence was used to observe the effect of four kinds of scaffolds on protein expression of tendon stem cells, $\operatorname{rod} 50 \mu \mathrm{m}$

The above results indicate that the modified TSA nanocomposite fiber has a stronger effect on promoting the differentiation of tendon system of tendon stem cells than the pure parallel nano-fibers. On the one hand, the stem cell nano-composite fibers combined with TSA provide physical induction factors for stem cell differentiation; on the other hand, they also provide chemical induction factors by releasing TSA. That is to say, TSA nano-composite fiber has synergistic effect in promoting the differentiation of tendon stem cells, which makes TSA modified nano-composite fiber have stronger biological induction activity.

Biomechanical test results were observed. The maximum tensile strength of tendon-bone tunnel interface in each group was shown in Table 1. At the same time point, the maximum tensile strength of tendon-bone tunnel interface between the experimental group and the control group was significantly different $(p<0.005)$. There were significant differences between the experimental group and the control group at three time points $(\mathrm{p}<0.005)$. The maximum tensile strength of BMP-2 group was higher than that of control group. There was no significant difference in the maximum tensile strength of the tendon-bone tunnel interface between the experimental group and the control group at the $2^{\text {nd }} \mathrm{W}$ after operation $(\mathrm{p}>0.005)$. At the $4^{\text {th }} \mathrm{W}$ after operation, there was a significant difference in the maximum tensile strength of the tendon-bone tunnel interface between the experimental group and the control group $(\mathrm{p}=0.005)$. The maximum tensile strength of tendon-bone tunnel interface was significantly different between the experimental group and the control group at the $8^{\text {th }} \mathrm{W}$ after operation $(\mathrm{F}=82.18, \mathrm{p}<0.005)$. At the $8^{\text {th }} \mathrm{W}$ after operation, there was a significant difference in the maximum tensile strength of the tendon-bone tunnel interface between the three groups $(\mathrm{F}=219.32$, $\mathrm{p}<0.005$ ). The maximum tensile strength of the tendonbone tunnel interface in the experimental group was $16.19,22.05$ and 48.67 percentage points higher than that in the control group at the $2^{\text {nd }}, 4^{\text {th }}$ and $8^{\text {th }} \mathrm{W}$ after operation, respectively. The results showed that the maximum tensile strength of the tendon-bone tunnel interface in the experimental group was significantly higher than that in the control group and the test values were significantly different.

\section{TABLE 1: MAXIMUM TENSILE STRENGTH}

\begin{tabular}{lcc}
\hline \multirow{2}{*}{ Team } & \multicolumn{2}{c}{ The maximum tensile strength $(\mathrm{N})$} \\
\cline { 2 - 3 } & $\begin{array}{c}\text { The experimental } \\
\text { group }\end{array}$ & $\begin{array}{c}\text { The control } \\
\text { group }\end{array}$ \\
\hline 2 w after operation & 58.49 & 50.49 \\
4 w after operation & 79.92 & 65.49 \\
8 w after operation & 114.9 & 77.29 \\
\hline
\end{tabular}

According to the same time point set by the experiment, the biomechanical test results of the interface stiffness of tendon-bone tunnel in each group showed that the stiffness of the experimental group was higher than that of the control group. There was a significant difference between the experimental group and the control group $(p<0.005)$. The results of the post-operative measurement of the interface stiffness of tendon-bone tunnel showed that there was a significant difference between the experimental group and the control group at the three set experimental time points $(p<0.005)$. There was no significant difference in the stiffness of tendon-bone tunnel interface between the experimental group and the control group at the $2^{\text {nd }} \mathrm{W}$ after operation $(p=0.205)$. There was no significant difference in the stiffness of tendon-bone tunnel interface between the experimental group and the control group at the $4^{\text {th }} \mathrm{W}$ after operation $(\mathrm{p}=0.057)$. At the $8^{\text {th }} \mathrm{w}$ after operation, 
there was a significant difference in the stiffness of tendon-bone tunnel interface between the experimental group and the control group $(\mathrm{p}<0.005)$. There was significant difference in the stiffness of tendon-bone tunnel interface between experimental group and control group at the $8^{\text {th }} \mathrm{W}$ after operation $(\mathrm{p}<0.005)$. The stiffness of tendon-bone tunnel interface in experimental group was $11.73,15.65$ and 50.59 percentage points higher than that in control group at 2,4 and $8 \mathrm{w}$ after operation (Table 2). At the $8^{\text {th }} \mathrm{W}$ after operation, the stiffness of tendon-bone tunnel interface in the experimental group was significantly higher than that in the control group. It was confirmed that the stem cell nano-composite fiber scaffold could enhance the bonding strength of tendon-bone tunnel interface, promote the repair of tendon-bone tunnel interface and improve the stiffness of tendon-bone tunnel interface in the experimental group.

\section{TABLE 2: RIGIDITY TABLE}

\begin{tabular}{lcc}
\hline \multirow{2}{*}{ Team } & \multicolumn{2}{c}{ The rigidity $(\mathrm{N} / \mathrm{mm})$} \\
\cline { 2 - 3 } & $\begin{array}{c}\text { The experimental } \\
\text { group }\end{array}$ & $\begin{array}{c}\text { The control } \\
\text { group }\end{array}$ \\
\hline 2 w after operation & 20.76 & 18.58 \\
4 w after operation & 25.73 & 22.24 \\
8 w after operation & 37.14 & 24.67 \\
\hline
\end{tabular}

The knee joint is the largest and most complex joint in the human body. The joint structure consists of the medial and lateral femoral condyle, the medial and lateral tibial condyle and the patella. It is a joint with more injury opportunities in the course of exercise. TSAmodified stem cell nano-composite fibers can guide cell morphological changes, while TSA can enhance the expression of Scx, a key transcription factor in tendon elevation. In addition, TSA-modified stem cell nanocomposite fibers significantly promoted the expression of other tendon-related genes at the level of gene and protein expression. Although the disordered scaffolds did not promote the differentiation of tendon cells, the expression of Scx and other tendon genes in cells could be significantly increased once the disordered scaffolds were modified by TSA, suggesting that TSA has a strong maintenance effect on the expression of tendon genes in tendon stem cells. However, the induction of tendon differentiation by TSA combined with stem cell nano-composite fibers is stronger than that by any single factor. The results of in situ tendon repair in vivo showed that TSA modified stem cell nano-composite fibers had the best effect on the repair of defective tendons. In addition to promoting the differentiation of tendon progenitor cells into tendon cells, materials also promote the formation of extracellular matrix of tendon and the maturation of collagen fibers. The main component of extracellular matrix of tendon is collagen I and the biomechanical properties are proportional to the parallelism and maturity of collagen fibers. The thickness of collagen fibers reflects the maturity of tendon. The tendon repaired by TSA modified stem cell nano-composite fibers is composed of collagen fibers of different sizes, which are different from other materials in ultrastructure. The distribution of collagen fibers was found to be bimodal. Although the diameter of collagen fibers was still different from that of normal tendons, the distribution pattern was similar and the mechanical properties were further improved. These results suggest that the active scaffold of histone deacetylase (HDAC) small molecule inhibitor stem cell nano-composite fibers can synergistically promote the differentiation and maturation of tendon system of tendon stem cells and better promote tendon regeneration ${ }^{[14]}$.

At present, most scholars believe that tendon stem cells can independently complete bone induction. Tendon stem cells have osteogenic induction effect and have been widely used in patients with nonunion and bone defect. Biological factors in tendon-bone tunnel interface healing after ligament reconstruction, experiments show that nano-fiber scaffolds can promote tendon-bone tunnel interface repair early after surgery ${ }^{[15]}$. Role of tissue engineering and stem cells in healing of tendon-bone tunnel interface after ligament reconstruction-current difficulties in repairing tendon-bone tunnel interface may help to solve them. The tendon tissue and bone tissue grow into healing according to artificial channels and the multi-phase scaffolds with gradient characteristics have different functional areas of different tissues. The role of bone-like materials in the healing of tendon-bone tunnel interface after ligament reconstruction: Bone-like materials are biologically active bone substitutes. Studies have shown that PCP may promote tendon healing in bone marrow canal in combination with calcium sulfate. Similarly, the physical factors of tendon-bone tunnel interface after ligament reconstruction still play an important role: low-intensity ultrasound can significantly promote the early repair of tendon-bone junction. The maximum pull-out strength of biomechanical test in the experimental group was higher than that in the control group at different periods. It is of great significance to the mechanical environment of tendon-bone structure restoration, which mainly promotes the healing of tendon in bone tunnel: good mechanical environment stimulation can promote the functional recovery of 
tendon-bone structure; conversely, inappropriate external mechanical loading has a negative impact on the restoration of tendon-bone structure, but the current research on the optimal mechanical environment for the repair of tendon-bone structure cannot explain. Although this experiment shows that stem cell nanocomposite fiber scaffolds promote tendon repair to a large extent, it provides a partial solution for the selection of seed cells and materials which are key to muscle bond regeneration. However, there are still some gaps in collagen fibers size and performance between repaired tendons and normal tendons, so future induction schemes need to be further explored ${ }^{[16]}$.

Starting from the source of seed cells and the maintenance of in vitro phenotype in tendon tissue engineering, based on the surface physical and topological structure of the matrix and the development of small chemical molecules, the phenotype maintenance and tendon differentiation of stem cells in vitro were optimized. In this experiment, nano-composite fiber scaffolds were used to reconstruct the tendon-bone tunnel interface during the reconstruction of ACL after sports injury. The results show that nano-composite fiber scaffolds have therapeutic effects on the recovery of sports ligament injury. The tendon-bone tunnel interface promotes the formation of the direct stop point after ACL reconstruction, improves the maximum tensile strength and stiffness of the tendon-bone tunnel interface and is conducive to the improvement of the pathophysiological characteristics and mechanical strength of the reconstructed ligament structure.

\section{Conflicts of interest:}

The authors declared no conflict of interest.

\section{REFERENCES}

1. Jiang YP, Yu R. Real-time inverse kinematics algorithm for motion modeling of human joint constraint. Automat Instrum 2018;230(12):202-4.

2. Proffen BL, Sieker JT, Murray MM, Akelman MR, Chin KE, Perrone GS, et al. Extracellular matrix-blood composite injection reduces post-traumatic osteoarthritis after anterior cruciate ligament injury in the rat. J Orthop Res 2016;34(6):9951003.

3. Garcia-Ruiz JP, Diaz Lantada A. 3D printed structures filled with carbon fibers and functionalized with mesenchymal stem cell conditioned media as in vitro cell niches for promoting chondrogenesis. Materials 2018;11(1):23.
4. Nyambat B, Chen CH, Wong PC, Chiang CW, Satapathy MK, Chuang EY. Genipin-crosslinked adipose stem cell derived extracellular matrix-nano graphene oxide composite sponge for skin tissue engineering. J Mater Chem B 2018;6(6):979-90.

5. Yang B, Zhao HQ, Zeng G, Zhong Y. DC capacitors voltage balancing strategy for cascaded STATCOM. J Power Supply 2016;14(5):128-36.

6. Xu Q, Yang GM, Qin HY, Bai JP. Effect of substrate bias on tribological properties of VC nano thin films. J Jilin Univ (Sci Ed). 2016;54(4):883-6.

7. Xue X, Sbragaglia M, Biferale L, Toschi F. Effects of thermal fluctuations in the fragmentation of a nanoligament. Phys Rev E 2018;98(1):012802.

8. Bargmann S, Soyarslan C, Husser E, Konchakova N. Materials based design of structures: Computational modeling of the mechanical behavior of gold-polymer nanocomposites. Mech Mater 2016;94:53-65.

9. Yu LY, Liu XF. Nano memory element to promote the electronic science and technology to achieve a major breakthrough. J China Acad Electron Inf Technol 2016;11(1):15-20.

10. Gerasimenko AY, Zhurbina NN, Kurilova UE, Ichkitidze LP, Selishchev SV, Suetina IA, et al. Knee joint ligament implants with composite nanocoatings. Biomed Eng 2016;50(3):206-9.

11. Yang F, Zhang JG, Hou YM, Zhang XD. Finite element simulation and optimization strategy of nanocomposites reinforced by inclusion with large aspect ratio. Comput Simul 2018;35(9):243-7.

12. Gao W, Wang Y, Basavanagoud B, Jamil MK. Characteristics studies of molecular structures in drugs. Saudi Pharm J 2017;25(4):580-6.

13. Gao W, Wang Y, Wang W, Shi L. The first multiplication atombond connectivity index of molecular structures in drugs. Saudi Pharm J 2017;25(4):548-55.

14. Yang BB, Li ZL, Wang JD. Preparation and electrochemical performance of nano $\mathrm{Si} / \mathrm{C}$ microsphere material. Chin J Power Sources 2017;41(10):1402-4.

15. Vollner F, Pilsl U, Craiovan B, Zeman F, Schneider M, Worner M, et al. Stability of knee ligament complex of Thielembalmed cadaver compared to in vivo knee. J Mech Behav Biomed Mater 2017;71:392-6.

16. Florida SE, VanDusen KW, Mahalingam VD, Schlientz AJ, Wojtys EM, Wellik DM, et al. In vivo structural and cellular remodeling of engineered bone-ligament-bone constructs used for anterior cruciate ligament reconstruction in sheep. Connect Tissue Res 2016;57(6):526-38.

This is an open access article distributed under the terms of the Creative Commons Attribution-NonCommercial-ShareAlike 3.0 License, which allows others to remix, tweak, and build upon the work non-commercially, as long as the author is credited and the new creations are licensed under the identical terms

This article was originally published in a special issue,

"Therapeutic Perspectives in Biomedical Research and Pharmaceutical Sciences and their Nursing Methods"

Indian J Pharm Sci 2021:83(4)Spl issue "235-242" 